\title{
Perspective
}

PERSPECTIVE Actualité en histoire de l'art

$2 \mid 2020$

Danser

\section{La céramique comme scène : des liens entre la danse et les vases grecs antiques}

The Ceramic Stage: Ways of Relating Ancient Greek Vases to Dance

Die Keramikbühne: Möglichkeiten der Inbezugsetzung von griechischen Vasen und Tanz

La scena della ceramica: modi per ripensare i legami tra le ceramiche della Grecia antica e la danza

La escena de la cerámica: maneras de pensar los lazos entre las cerámicas de la antigua Grecia y la danza

\section{Tyler Jo Smith}

Traducteur : Étienne Gomez

\section{(2) OpenEdition}

Journals

Édition électronique

URL : https://journals.openedition.org/perspective/20490

DOI : $10.4000 /$ perspective. 20490

ISSN : 2269-7721

Éditeur

Institut national d'histoire de l'art

Édition imprimée

Date de publication : 30 décembre 2020

Pagination : 109-120

ISBN : 978-2-917902-90-5

ISSN : $1777-7852$

Référence électronique

Tyler Jo Smith, «La céramique comme scène : des liens entre la danse et les vases grecs antiques », Perspective [En ligne], 2 | 2020, mis en ligne le 30 juin 2021, consulté le 29 juillet 2022. URL : http:// journals.openedition.org/perspective/20490; DOI : https://doi.org/10.4000/perspective.20490 


\title{
La céramique comme scène : des liens entre la danse et les vases grecs antiques
}

\author{
Tyler Jo Smith
}

\section{Une scène céramique?}

La danse et les vases grecs antiques entretiennent des rapports complexes. De nombreux vases fabriqués dans la ville d'Athènes et dans d'autres centres, aux VI ${ }^{e}$ et $V^{e}$ siècles avant notre ère (époques archaïque et classique), développent une iconographie de la danse. En théorie, ces images, qui apparaissent sur des vases présentant des formes très variées et fabriqués selon des techniques artistiques différentes, devraient avoir beaucoup à nous apprendre sur les manières de danser et sur les circonstances dans lesquelles on dansait dans la culture grecque antique. En réalité, les vases (en tant qu'objets matériels) et leur ornementation figurée (en tant que témoignage visuel) fonctionnaient comme un tout dans un grand nombre de contextes, servant les vivants comme les morts et représentant des aspects de la vie quotidienne comme de l'imaginaire. En un mot, il était impossible de réaliser l'adéquation entre ornementation bidimensionnelle du vase en céramique et mouvement tridimensionnel du corps qui danse, et peut-être aucun artiste n'a-t-il jamais réellement visé ce but. Néanmoins, les études consacrées à la danse telle qu'elle est figurée sur les vases ont une histoire quasiment aussi longue que les études formelles concernant les vases peints eux-mêmes, et les spécialistes s'interrogent depuis plus d'un siècle à propos de la valeur et de la fiabilité de ces images en tant que documents sur la danse antique ${ }^{1}$. Malgré une meilleure compréhension de la production de vases (grâce à de nouvelles découvertes et à des progrès scientifiques), et une évolution des méthodes de l'analyse iconographique, si l'intérêt pour la danse grecque antique est toujours aussi vif, il n'y a pas de consensus sur la meilleure manière de réconcilier ces deux objets d'étude.

Ce bref article s'intéresse à la relation entre la danse grecque antique, un sujet qui nous est connu par des sources textuelles et visuelles, et les vases des époques archaïque et classique comportant des scènes de danse. Il présente certaines des interprétations et des utilisations passées et présentes de la danse sur les vases, ainsi que certaines perspectives plus récentes permettant d'envisager à la fois la danse et les vases. Il s'agit donc de reconnaître l'existence d'attitudes parallèles : d'un côté, une tendance datée à la reconstitution de la 


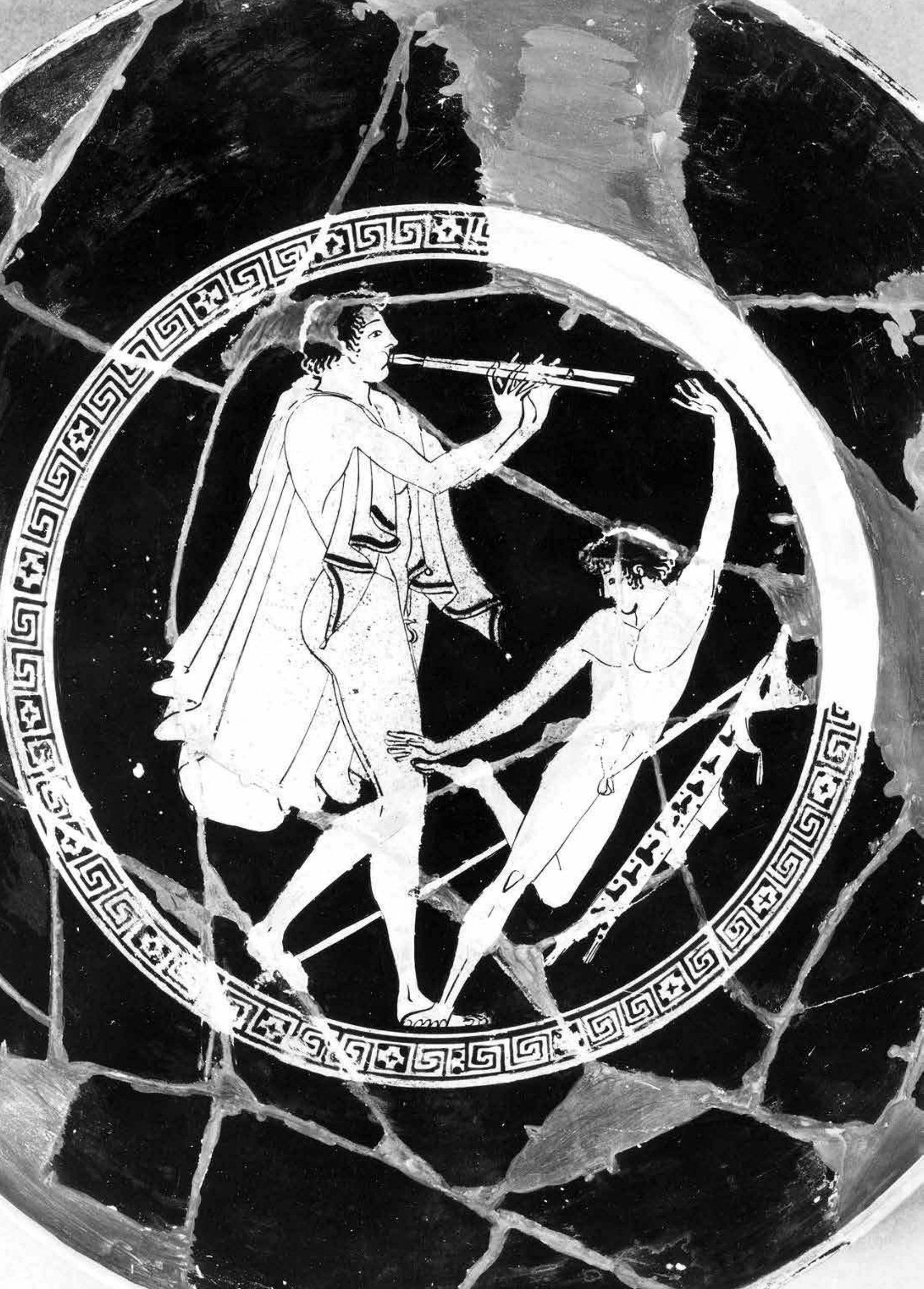




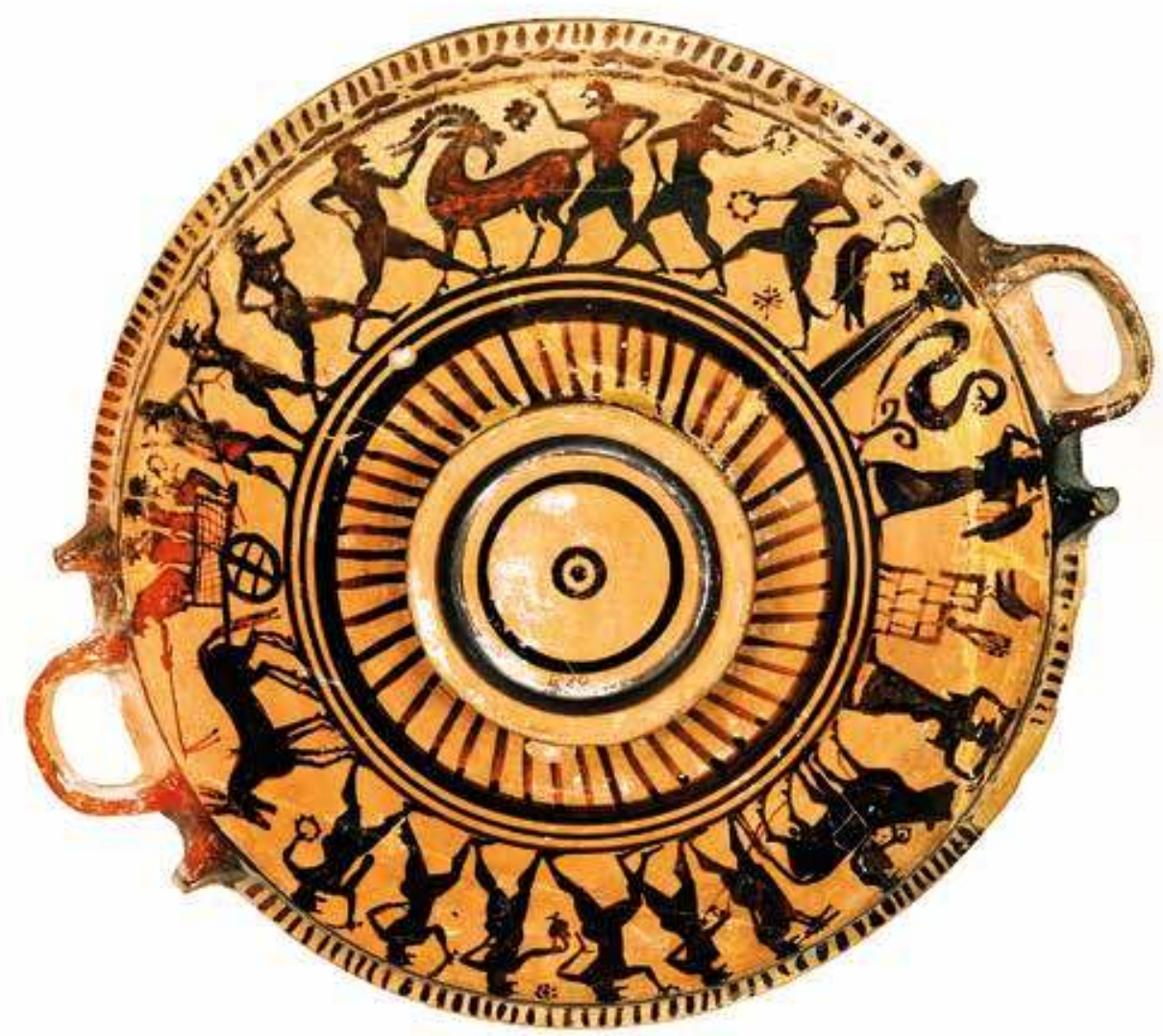

danse grecque antique, une forme artistique éphémère, $\mathrm{d}$ 'après les représentations attestées sur les vases (ainsi que dans d'autres sources artistiques) ; de l'autre, des tentatives plus récentes de lier danse et vases en les traitant comme des sujets d'étude en soi et comme des types de témoignages en lien l'un avec l'autre. À la lumière des textes et des images, il apparaît clairement que la danse 1. Peintre de Triptolème, komos avec deux jeunes danseurs, intérieur d'un vase athénien à figures rouges, vers 480 avant J.-C., Paris, musée du Louvre.

2. Procession religieuse avec danseurs, lekanis béotienne à figures noires, milieu du VI siècle avant J.-C., Londres, British Museum. était liée à de nombreux aspects de la culture grecque antique, de la vie quotidienne à la mythologie, du culte aux fêtes religieuses, des représentations publiques aux représentations privées. Une telle situation fait de l'iconographie de la danse sur les vases un cas unique, contraignant les spectateurs d'aujourd'hui à envisager différents contextes possibles derrière les représentations qui ont subsisté ${ }^{2}$.

Il est ici nécessaire d'expliquer certaines des stratégies déployées par les peintres pour représenter les figures de danseurs sur la surface des céramiques, en soulignant à quel point les formes de vases et les techniques picturales associaient cette expérience de la danse à un contexte social donné. Prenons par exemple un kylix à figures rouges fabriqué à Athènes au début du V siècle avant notre ère (fig. 1), dont la face intérieure représente une scène de komos (cortège festif) avec deux jeunes hommes imberbes. Une telle forme était probablement destinée à la consommation de vin lors d'un symposion, le banquet antique (réunissant principalement des hommes), où la danse pouvait intervenir comme spectacle (mobilisant des hommes et des femmes), comme activité, ou les deux. Prenons maintenant une lekanis (coupe plate) à figures noires, fabriquée en Béotie au milieu du $\mathrm{VI}^{\mathrm{e}}$ siècle (fig. 2), où des danseurs de sexe masculin apparaissent dans une 


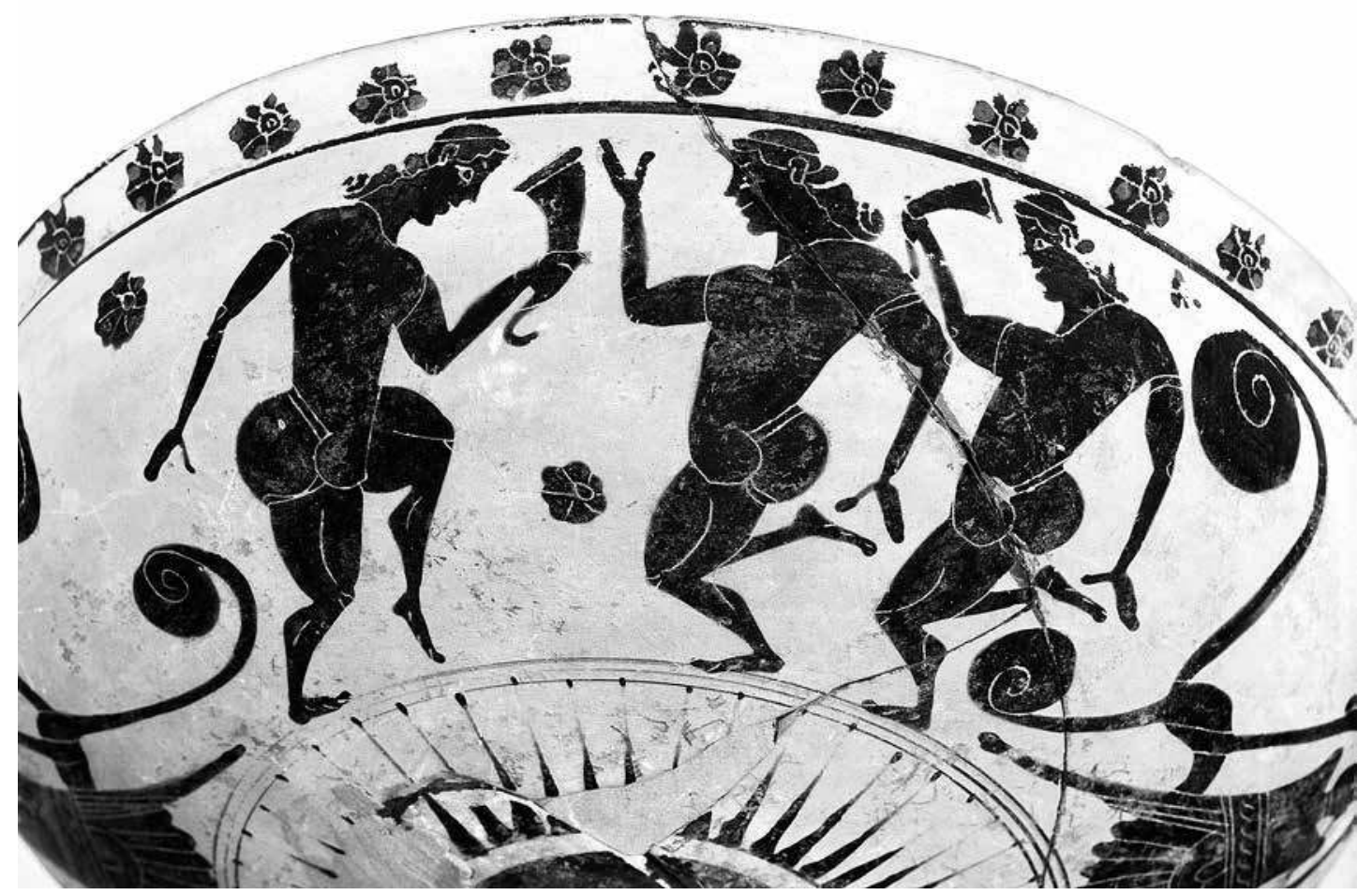

3. Groupe des Comastes, comastes tenant une corne à boire (rhyton), coupe athénienne à figures noires, vers 575 avant J.-C., Paris, musée du Louvre. composition complexe avec plusieurs figures. Ce choix de forme, combiné au recours inhabituel à la technique de la céramique à figures noires, suggère que le vase devait servir lors d'une fête religieuse ou être inhumé avec un défunt ${ }^{3}$. Dans les deux cas, pour interpréter

la relation entre danse et vase, il faut prendre en compte l'image peinte mais aussi la forme de vase choisie et la technique décorative utilisée par l'artiste. La détermination des lieux d'où viennent ces vases individuels, et ce qu'ils suggèrent de leur destination / fonction et de leurs utilisateurs / spectateurs, du point de vue, entre autres, de la danse, représente un problème archéologique supplémentaire. L'hypothèse selon laquelle les scènes de danse peintes sur les vases retrouvés dans des tombes ou des sanctuaires antiques étaient toujours inspirées de danses funéraires ou cultuelles et destinés à être inhumés avec un défunt précis, ou consacrés à une divinité particulière, n'est pas confirmée par les vases qui ont subsisté, qu'ils aient été découverts sur des sites grecs ou non, et qu'ils aient été fabriqués à Athènes ou dans d'autres lieux. Naturellement, on trouve toujours des exceptions, comme les krateriskoi retrouvés dans le sanctuaire d'Artémis à Brauron, montrant des jeunes filles qui prennent part à des rituels d'initiation (dont des danses) à cet endroit et en l'honneur de cette déesse ${ }^{4}$.

Comment, dès lors, regarder les vases grecs antiques comme des témoignages de phénomènes culturels, parmi lesquels la danse et d'autres spectacles? Leur étude représente une entreprise holistique. Comme le montrent de nombreux exemplaires signés, ils étaient tournés par un potier et décorés par un peintre, même si, dans certains cas, les deux tâches étaient accomplies par une même personne ou par les membres d'un même atelier. Un vase, indépendamment de sa qualité ou de son lieu de découverte, doit néanmoins être considéré sous plusieurs perspectives : non seulement comme la somme des éléments 
qui le constituent, mais aussi comme le reflet de l'époque, de l'endroit et des artistes qui l'ont produit. Les archéologues spécialisés considèrent ces objets de belle facture comme des artéfacts aussi bien matériels que visuels, qui doivent être envisagés en fonction de leurs qualités esthétiques formelles (ce qui inclut les motifs non figuratifs) de même que pour leur nature et leur matérialité tactiles. Indépendamment de leur sujet ou de leur échelle, ils étaient faits pour être vus, utilisés et manipulés. Si l'étude et l'observation approfondies des vases à figures noires et à figures rouges, ou exécutés selon d'autres techniques, peut nous aider à mieux comprendre la danse grecque antique, les vases représentant des scènes de danse doivent être envisagés en tant que tels, et leurs limites en tant que documents doivent toujours être prises en compte. Lorsqu'on s'appuie sur des vases dans l'étude des activités quotidiennes (par opposition aux récits mythologiques) - qu'il s'agisse de la danse ou du théâtre, du culte religieux, de la course, de la chasse et de la pêche, etc. -, il est très important de ne pas oublier que ces images ne sont pas des instantanés photographiques de la vie à Athènes, à Corinthe, à Sparte ou ailleurs. Une scène de danse sur un vase ne doit tout simplement pas être considérée comme la représentation d'un moment figé dans le temps. Si les peintres de vases avaient sans doute des connaissances sur la danse, que ce soit par l'observation ou par leur expérience personnelle, ils suivaient invariablement les conventions de leur art. Il me semble que, la plupart du temps, ce qu'ils voulaient fixer, ce n'étaient ni les pas et les poses, ni les mouvements et la gestuelle d'une danse donnée, mais son concept et son contexte.

Indépendamment des questions, des approches et des perspectives qui ont permis aux spécialistes d'envisager conjointement danse et vases au fil du temps, différenciant les styles de danse et les types de danseurs représentés - je résumerai brièvement ces points ci-dessous -, nous devons aujourd'hui donner la priorité à l'étude approfondie des vases en tant que tels, d'un point de vue visuel aussi bien que matériel, individuel aussi bien que collectif. Comment les peintres de vases représentaient-ils la danse avec les outils dont ils disposaient ? Comment pouvaient-ils la traduire et la communiquer visuellement? S'il s'agit d'une question complexe qui recouvre de multiples aspects, deux points ont déjà été mentionnés : la forme et la technique. Pour les peintres de vases, apposer une ornementation figurée sur une surface incurvée représentait d'emblée de nombreux défis. Il apparaît toutefois clairement que certains d'entre eux (quoique pas tous) étaient spécialistes d'une technique, de la décoration de certaines formes de vases et d'une iconographie précise. Ainsi, les peintres du groupe dit des Comastes décoraient le plus souvent une forme de coupe spécifique (fig. 3) avec des figures de noceurs dansant dans un komos, eux-mêmes appelés " comastes $^{5}$ ". Ces coupes sont toujours décorées sur leur face extérieure, et les peintres placent opportunément les fessiers exubérants des danseurs à l'endroit le plus incurvé de la coupe, incitant le spectateur, en regardant mieux, à remarquer la claque sur les fesses qui était leur geste signature. Les comastes peuvent nous paraître un peu étranges avec leurs corps représentés de profil, leurs mains effilées et leurs postures figées. Tous ces aspects découlent cependant de la technique de la figure noire, un système plat et bidimensionnel qui ne permet pas d'animer le corps humain de manière convaincante. À titre de comparaison, revenons à la coupe à figures rouges mentionnée plus haut (fig. 1), où les figures de danseurs apparaissent sur la face intérieure et où une stratégie artistique différente a été déployée pour occuper l'espace, parfaitement circulaire. La technique plus avancée de la céramique à figures rouges a par ailleurs permis au peintre de mettre les danseurs en mouvement et de superposer figures et objets d'une manière esthétiquement agréable. Le contraste entre ces deux coupes ne tient pas uniquement à une question de choix de représenter tel ou tel moment de danse. Les formes des vases et les techniques de fabrication ont inspiré la représentation tout autant que les participants, leurs poses et leurs gestes. 


\section{Questions / approches / perspectives : passé et présent}

Les études sur la danse et les vases grecs antiques exigent une connaissance générale des questions et des approches, directes aussi bien qu'indirectes, qui orientent traditionnellement l'analyse. Contrairement aux autres aspects des études portant sur la peinture sur vases, la danse occupe une place tout à fait singulière et attire l'attention de gens très différents : les danseurs, les acteurs, les professeurs de danse, les metteurs en scène, les chorégraphes, ou encore les historiens de la danse et les spécialistes de l'Antiquité. Immanquablement, ces différents groupes envisagent la relation entre danse et vases selon des angles de vue différents, au service de leurs besoins propres. On part généralement du compositeur et musicologue français Maurice Emmanuel (1862-1938) qui, dans La Danse grecque antique d'après les monuments figurés (1896), s'efforça de reconstituer les mouvements des chorégraphies antiques sur la base d'une interprétation littérale des arts visuels. Son livre abondamment illustré, qui eut de nombreux lecteurs mais aussi de nombreux défenseurs et héritiers (parmi lesquels Louis Séchan, Germaine Prudhommeau et Marie-Hélène Delavaud-Roux), exerça une profonde influence sur les conceptions de la danse grecque antique jusqu'au milieu du XX $X^{e}$ siècle, et sur les danseurs et les chorégraphes qui en eurent connaissance ${ }^{6}$. Sa tentative de reconstituer les chorégraphies antiques et d'en rapprocher le ballet à la fin du XIX ${ }^{e}$ siècle, quoique très largement appuyée sur les vases, était inspirée par une analyse chronophotographique du corps humain. Si une telle perspective parait datée, l'affirmation récente selon laquelle « les archéologues Edmond Pottier et Maxime Collignon guidèrent Emmanuel dans l'histoire de l'art antique et l'aidèrent à affiner son œil artistique " nous permet d'envisager dans une perspective plus académique ses méthodes de reconstitution de la danse sur la base de l'iconographie ${ }^{7}$.

Les danseurs et les professeurs de danse jouèrent aussi un rôle dominant dans ces premières associations entre danse et vases. Le cas le plus célèbre est celui de la danseuse américaine Isadora Duncan (1877-1927), qui trouva dans les vases grecs antiques une source d'inspiration pour ses chorégraphies et sa routine quotidienne. En partisane de la danse naturelle, elle cherchait davantage à émanciper les mouvements corporels et les vêtements associés des contraintes du passé. Comme je l'ai exposé ailleurs, Isadora et son frère Raymond étudièrent les collections de vases des plus importants musées $\mathrm{d}^{\prime}$ Europe $^{8}$. Si elle ne reconstitua pas littéralement les chorégraphies antiques, Isadora redonna vie aux poses et aux gestes observés sur les vases - transposant, en somme, la scène céramique dans la modernité 9 . Eva Palmer (ultérieurement Sikelianos ; 1874-1952), une actrice américaine qui portait parfois, comme ses proches Isadora et Raymond, le costume " grec » antique (inspiré par les vases et les découvertes archéologiques), vécut un certain temps en leur compagnie à Athènes. Principalement intéressée par le théâtre, elle cherchait à reconstituer la scène théâtrale, comme en témoigne le festival delphique qu'elle organisa en 1927 avec son mari (le poète grec Angelos Sikelianos) - elle y mit en scène une tragédie grecque, avec des chœurs et des costumes tissés à la main, et les ruines de la Delphes antique pour décor ${ }^{10}$. Madame Mariquita, la chorégraphe de la danseuse et actrice française Régina Badet (1876-1949), adopta quant à elle une approche différente, qu'elle décrivit ainsi en 1908 dans un entretien : " Je me suis aussitôt empressée de visiter des musées, j'ai regardé des vases antiques, des fresques, des statues... et dans des documents longuement étudiés, examinés avec soin, j'ai trouvé des poses, des attitudes, des gestes, sur quoi reposera tout mon divertissement ${ }^{11}$. " Parmi ceux qui ont cherché à pratiquer et à renouveler la danse moderne par la fréquentation de la culture visuelle et littéraire grecque antique, on trouve aussi des figures comme le chorégraphe russe Michel Fokine (1880-1942) et la danseuse Anna Pavlova (1881-1931), qui avaient étudié l'art 
grec dans les musées et qui furent liés tous les deux aux Ballets russes, eux-mêmes souvent inspirés par l'art grec ; Ruby Ginner (18861978), l'auteur britannique de The Revived Greek Dance: its Art and Technique (1933), un livre illustré de vases peints d'origine athénienne ; et la chorégraphe américaine Martha Graham (1894-1991), qui puisa abondamment dans la mythologie et les thèmes classiques $^{12}$. On peut également signaler ici la récente exposition sur les Ballets russes qui s'est tenue à l'Institute for the Study of the Ancient World de la New York University, et dont le catalogue intégrait des vases grecs à des analyses sur l'histoire, le costume, la gestuelle et la chorégraphie ${ }^{13}$.

Les spécialistes de l'Antiquité et les historiens de la danse s'intéressent aussi aux liens entre danse et vases au fil des siècles. Leurs études considèrent souvent les vases comme des témoignages confirmant l'existence de danses attestées dans les écrits antiques, ou comme des représentations de danses réelles.

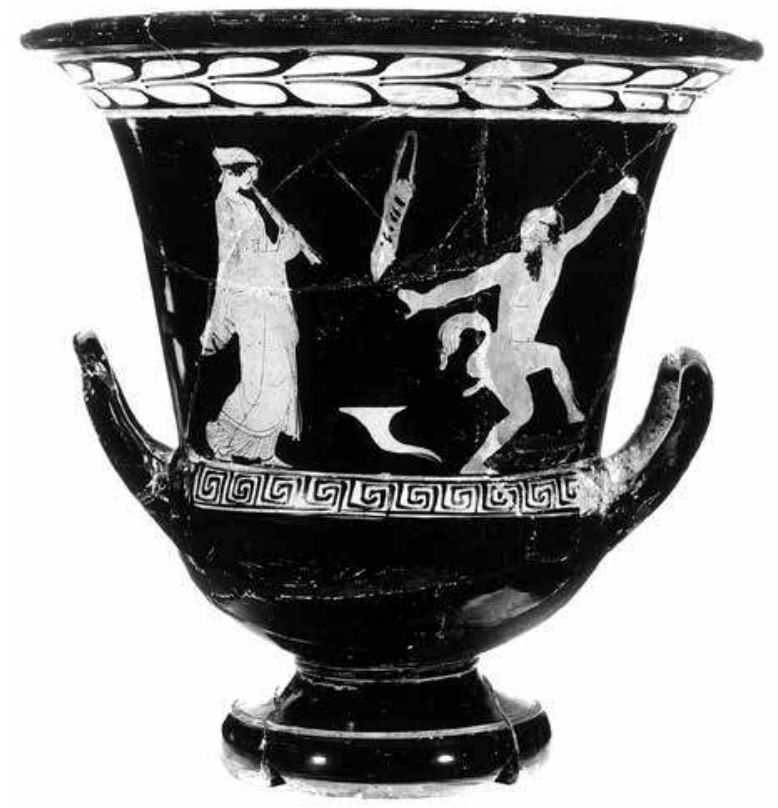

4. Satyre dansant et figure féminine (ménade ?) jouant de la flûte, avec une corne à boire au sol, calicecratère athénien à figures rouges, milieu du VI ${ }^{\mathrm{e}}$ siècle avant J.-C., Paris, musée du Louvre. Au XXe siècle, c'est à la chercheuse américaine Lillian B. Lawler (1898-1990) que l'on doit la contribution la plus nourrie de ce point de vue, avec toute une série d'articles et un ouvrage de référence, The Dance in Ancient Greece (1964), dont les conclusions, discutables, reposaient largement sur une interprétation des vases en tant que documents attestant de spectacles, de techniques et de danses précises ${ }^{14}$. Malgré leur ancienneté, les publications de Lawler doivent être mentionnées ici en tête de liste, du fait que toute la critique ultérieure, jusqu'à aujourd'hui, est inévitablement amenée à les fréquenter. Ensuite, le critique le plus fiable et le plus aguerri sur la danse grecque antique est Frederick G. Naerebout, auteur du livre Attractive Performances. Ancient Greek Dance: Three Preliminary Studies (1997) ainsi que de nombreux articles sur l'histoire, la documentation et les méthodes de recherche; dans nombre de ses travaux, il a développé des analyses minutieuses de représentations artistiques, parmi lesquelles des vases, tout en remettant régulièrement en question la validité du recours aux images (aux " sources non écrites ") en tant que documents historiques ${ }^{15}$.

D'autres spécialistes, à la fin du $\mathrm{XX}^{\mathrm{e}}$ siècle, associent vases et textes littéraires dans le cadre d'études sur la danse grecque antique et les domaines apparentés, mais leurs contributions sont inégales et l'on y chercherait en vain une vision, une base théorique ou une approche homogènes. L'archéologue antique Frank Brommer se contente ainsi d'envisager le problème d'un point de vue général et de regrouper les vases en catégories correspondant aux danses répertoriées et connues par des témoignages littéraires, comme le geranos (danse de la grue) et la sikinnis (danse des satyres), sans fournir autre chose que des analyses minimales ${ }^{16}$. Le spécialiste de la littérature grecque antique Steven Lonsdale, abordant le thème de la danse et de la religion, puise dans les représentations artistiques pour " élargir le témoignage de l'écriture " et considère les vases comme " les objets centraux des danses rituelles », fonctionnant $\mathrm{d}^{\prime}$ « une manière auto-référentielle ${ }^{17}$ ». Dans 
une imposante annexe à son étude pionnière sur les acteurs antiques (principalement étayée sur les inscriptions), Paulette Ghiron-Bistagne présente quant à elle l'iconographie de la danse sur les vases comme une source majeure de témoignages sur les acteurs et les origines du théâtre, suivant en cela la tradition établie par les chercheurs et les archéologues qui s'étaient attachés à ces sujets avant elle ${ }^{18}$. Les travaux sur les danses pyrrhiques de Jean-Claude Poursat, Marie-Hélène Delavaud-Roux et Paola Ceccarelli traitent tous les vases comme une source majeure de témoignages, mais leur stratégie mélange iconographie et reconstitution d'une part, initiation et technique d'autre part ${ }^{19}$. Pour retracer l'histoire de la danse grecque antique au fil des siècles, de l'Antiquité jusqu'à nos jours, il faut également mentionner, inspirés par un intérêt authentique pour la pratique et le spectacle, les travaux et les efforts de vulgarisation réalisés à Athènes par le Dora Stratou Dances Theatre ${ }^{20}$. Plusieurs ouvrages récents, en l'occurrence des actes de colloques organisés par d'autres groupes, proposent également des contributions liant danse et vases grecs antiques dans le cadre d'études sur la musique, la gestuelle, les spectacles ou encore l'archéologie ${ }^{21}$. Si ces ouvrages apportent sans doute la preuve la plus nette de la multiplicité des questions, des approches et des perspectives les plus novatrices sur nos sujets d'étude communs, ils témoignent collectivement du défaut d'accord qui, encore aujourd'hui, mine toute possibilité de lier danse et vases d'une manière poursuivie et cohérente.

Les spécialistes des vases peints, à l'opposé, traitent l'iconographie de ceux-ci comme un témoignage sur la danse antique, ses décors et ses exécutants. Le débat, vieux de plusieurs décennies, sur le "danseur rembourré ", le comaste stéatopyge que l'on retrouve souvent sur les vases archaïques du $\mathrm{VI}^{\mathrm{e}}$ siècle à figures noires, sources de la majeure partie des peintures sur vases représentant des scènes de danse parvenues jusqu'à nous (fig. 3), se prolonge dans de nombreux ouvrages et articles. Des hypothèses récentes tendent à s'affranchir de l'interprétation classique qui en fait une figure du culte dionysien ou un acteur de proto-théâtre, le présentant tantôt comme un danseur intervenant dans une diversité de contextes allant du symposion aux fêtes religieuses (fig. 2), tantôt comme une figure comique ou parodique, revêtant un sens littéraire ou politique ${ }^{22}$. Il faut signaler en particulier le cas des comastes peints avec des pieds difformes (fig. 5), peut-être à l'image d'Héphaïstos, le dieu de la forge, frappé de claudication ${ }^{23}$. Un certain nombre d'études importantes puisent cependant des deux côtés,

5. "Danseur rembourré " au pied difforme, reconstitution (dessin: D. Weiss) d'un fragment d'une oinochoé corinthienne, début du $\mathrm{VI}^{\mathrm{e}}$ siècle avant J.-C. (Corinthe, Musée archéologique).

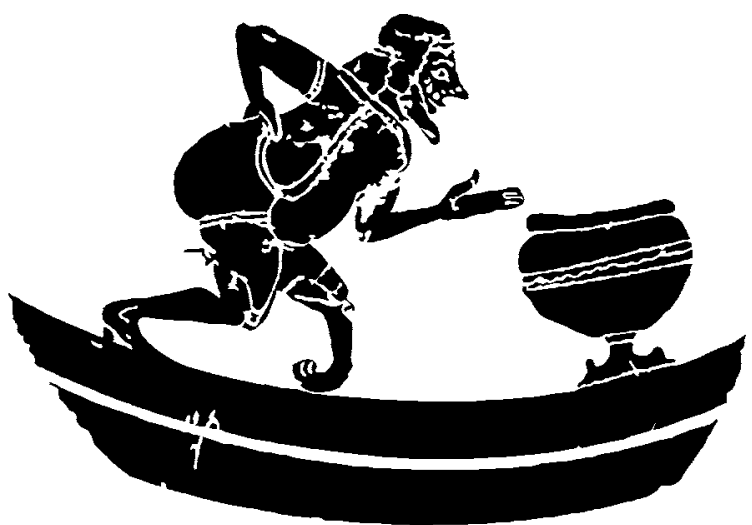
intégrant les comastes dansants (céramique à figures noires ou à figures rouges [fig. 1 et 3]) à des développements sur Dionysos, le chœur dramatique ou le symposion ${ }^{24}$. Parallèlement, les figures de comastes - et autres danseurs mortels - peintes sur des vases athéniens, corinthiens et laconiens, sont associées par des chercheurs divers, selon le contexte, aux chours animaux, aux dithyrambes, à la musique, aux satyres dansants (fig. 4), aux drames satyriques et au travestissement. De telles études ne font que souligner le rôle fondamental, quoique controversé, de ces figures pour l'analyse et l'interprétation de la danse sur vases dans les différentes contrées de la Grèce, et de la place de la danse dans la culture grecque d'une manière générale ${ }^{25}$. 


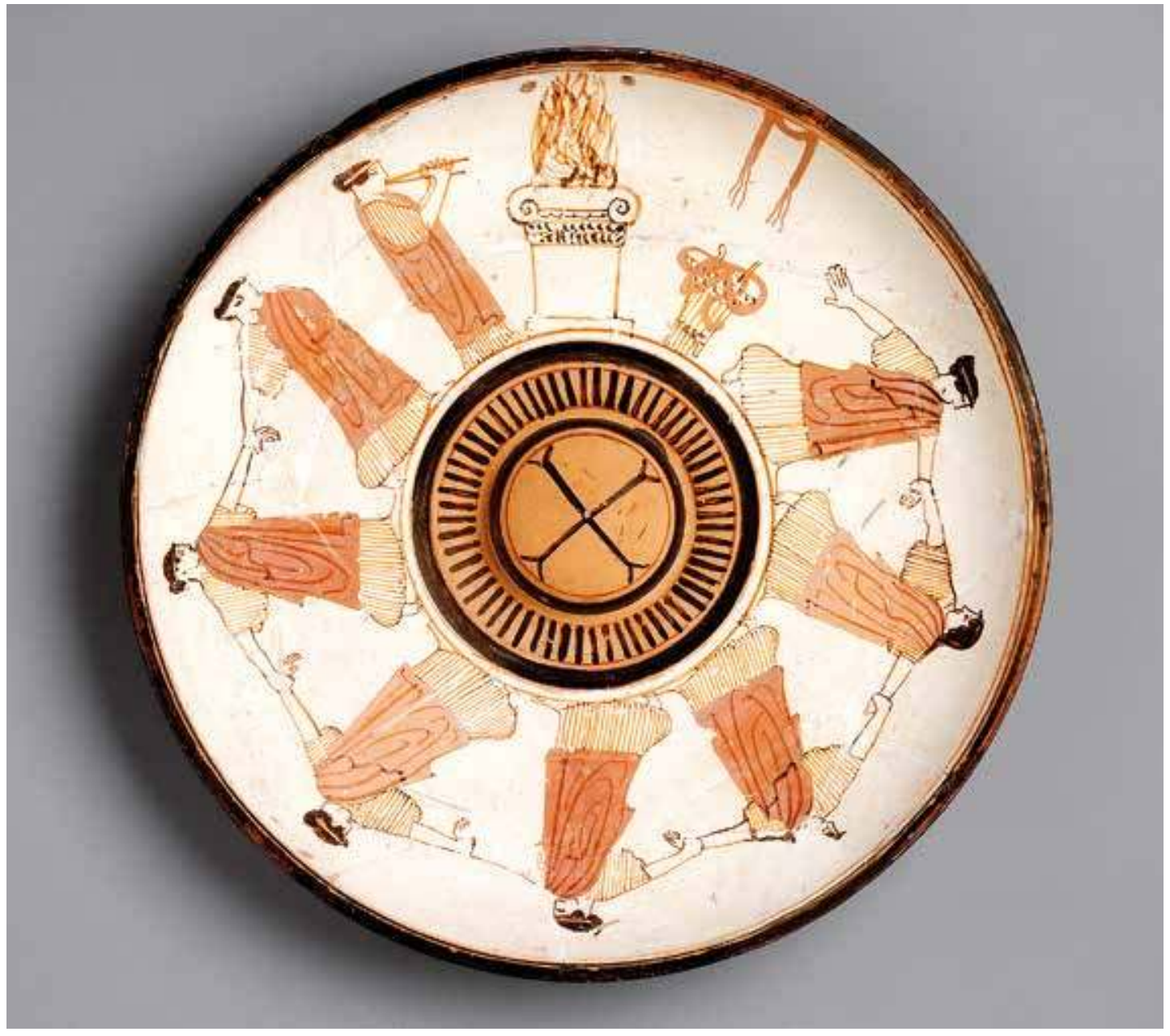

Une autre catégorie importante et largement débattue de scènes de danse sur vases, dont les témoignages sont nombreux, est celle qui représente des femmes. Deux catégories principales se distinguent en fonction de la 6. Peintre de Londres D12, femmes dansant en direction d'un autel, phiale athénienne, céramique blanche, vers 450 avant J.-C., Boston, Museum of Fine Arts, fonds Edwin E. Jack. qualité des danseuses et du style de mouvement, et elles se prêtent généralement à des analyses différentes ${ }^{26}$. Les peintures de danseuses exécutant un mouvement répétitif, en se tenant par le bras ou par la main, apparaissent dans la période géométrique tardive (env. 750-700 avant J.-C.), et cette iconographie frappe par sa longévité. Ces images sont associées soit aux funérailles d'élite (funérailles homériques ?), soit aux fêtes en l'honneur de dieux et de déesses ${ }^{27}$. Une phiale (coupe pour les libations) fabriquée à Athènes au milieu du $\mathrm{V}^{\mathrm{e}}$ siècle en donne un exemple particulièrement marquant, où des femmes parées avec art composent par leur chaîne une formation circulaire à l'intérieur de la coupe, elle-même circulaire (fig. 6). Elles dansent selon un pas convenu, en se tenant par le poignet et en se déplaçant ensemble au son d'une flûte. Leur but est un autel illuminé, où une phiale exactement comme celle-ci devait servir aux libations à la divinité : la forme, l'iconographie et la fonction constituent ainsi un tout cohérent. À rebours, de nombreux vases athéniens associés à la consommation de vin, 
comme la coupe ou le krater (bol mélangeur), figurent des ménades ou des nymphes dansantes, souvent rejointes par des satyres (fig. 4) et parfois accompagnées par Dionysos, le dieu du vin, en personne. L'ambiguïté inhérente à ce genre de scènes, dont on ne sait si elles représentent des épisodes mythologiques ou des créatures mortelles, fait qu'il est difficile de savoir qui ces femmes sont censées incarner ${ }^{28}$. Au cœur du groupe le plus énigmatique se trouvent les vases dits "des Lénéennes ", associés au culte de Dionysos dans la ville d'Athènes, qui représentent des femmes dansant de manière frénétique et versant du vin contenu dans un stamnos (vase rituel pour le service du vin) autour de l'effigie du dieu ${ }^{29}$. L'hypothèse d'une fonction rituelle est renforcée par le choix, de la part des peintres, du stamnos en tant que forme de vase adéquate pour mettre en scène cette iconographie singulière de la danse dans la céramique à figures rouges.

\section{Prochaines étapes?}

Malgré ces nombreuses tentatives - en apparence fructueuses - d'étudier les liens entre vases grecs, avec leurs formes et leurs techniques diverses, et danse antique, soit comme réalité, soit comme reflet, il reste de nombreuses pistes à explorer. Archéologues classiques et historiens de l'art doivent d'abord accorder une attention plus rigoureuse non seulement aux contextes culturels dans lesquels ont été employés les vases illustrant des scènes de danse, mais aussi aux contextes archéologiques précis dans lesquels ils ont été découverts ${ }^{30}$. Les études comparées avec les céramiques peintes dans d'autres régions du monde, comme en Amérique du Sud où une culture matérielle similaire a été mieux contextualisée (sur le plan culturel comme archéologique), peuvent dessiner de nouvelles possibilités pour l'étude des vases grecs antiques en tant que témoignages d'une activité rituelle attestée comme la danse ${ }^{31}$. Dans le même ordre d'idées, les progrès de l'archéologie sensorielle devraient permettre d'associer un sujet multi-sensoriel comme la danse à des objets multi-sensoriels comme les vases ${ }^{32}$. De nombreux vases ne représentent-ils pas des danseurs eux-mêmes équipés de vases comme des rhytons, ou cornes à boire (fig. 3 et 4) ? Enfin, une collaboration à la fois plus suivie et plus approfondie entre spécialistes et praticiens de la danse - sous la forme de séminaires et d'ateliers comme ceux que développe déjà, à Athènes, l'Ancient Orchesis Study Group (Dora Stratou Dances Theatre) - représenterait une opportunité d'aborder de nouvelles questions, d'introduire de nouvelles idées et d'écrire de nouvelles histoires. Au lieu de danseurs qui utilisent les vases pour reconstituer la danse antique et de chercheurs qui tentent d'identifier et de nommer des routines de danse disparues, la collaboration entre savoir scientifique et pratique de la danse permettra peut-être à chacun d'ouvrir de nouvelles perspectives, de révéler un potentiel insoupçonné, et de renouveler les liens entre les vases grecs et les nombreuses facettes de la danse antique ${ }^{33}$.

Cette contribution a été traduite de l'anglais par Étienne Gomez. 


\section{Tyler Jo Smith}

Tyler Jo Smith est professeure d'art classique et d'archéologie, directrice du Programme archéologique interdisciplinaire à l'University of Virginia. Spécialiste de la peinture et de l'iconographie des vases grecs, elle travaille essentiellement sur les images de la performance, en particulier de la danse, du théâtre et de la religion. Elle est l'auteure de Komast Dancers in Archaic Greek Art (Oxford / New York, Oxford University Press, 2010), et a codirigé A Companion to Greek Art (avec Dimitris Plantzos, Malden, Mass. / Oxford, Wiley-Blackwell, 2012-2018), et Religion in the Art of Archaic and Classical Greece (Philadelphia, University of Pennsylvania Press, 2021). Elle écrit un livre sur les vases grecs du Sir John Soane's Museum, à Londres.

\section{NOTES}

Je remercie Judith Delfiner et Marie Caillat pour leur intérêt et leur aide dans la rédaction de cet article. J'ai présenté certains aspects de ma recherche à Athènes, au $54^{e}$ Congrès mondial de recherche en danse (juillet 2019) et à l'Ancient Orchesis Study Group of the Dora Stratou Dances Theatre (mars 2019), ainsi qu'à Paris, en tant que Professeure invitée à l'École des hautes études en sciences sociales (EHESS) au printemps 2017.

1. Frederick G. Naerebout, Attractive Performances. Ancient Greek Dance: Three Preliminary Studies, Amsterdam, Giebern, 1997, première partie ; Brian A. Sparkes, The Red and the Black: Studies in Greek Pottery, Abingdon, Routledge, 1996.

2. La représentation de la danse dans d'autres types de sources dépasse le cadre de cet article. On trouvera des exemples, notamment des terres cuites, des figurines de bronze et des sculptures en relief dans Frederick G. Naerebout, "Achoreutos apaideutos: Dance in Ancient Greece », dans Clare Fitzgerald (dir.), Hymn to Apollo: The Ancient World and the Ballets Russes, Princeton, Princeton University Press, 2019, p. 30-51 ; et Frederick G. Naerebout, "The Baker Dancer and other Hellenistic Statuettes of Dancers: Illustrating the Use of Imagery in the Study of Dance in the Ancient Greek World ", dans Imago Musicae, vol. 18-19, 2001-2002, p. 59-83.

3. Tyler Jo Smith, Komast Dancers in Archaic Greek Art, Oxford, Oxford University Press, 2010, p. 173-174.

4. Tyler Jo Smith, Religion in the Art of Archaic and Classical Greece, Philadelphia, University of Pennsylvania Press, 2021, ch. V, fig. 170 a-b.

5. Smith, 2010, cité n. 3, ch. 2.

6. Naerebout, 1997, cité n. 1, p. 73-75, 85, 112-113 ; Samuel N. Dorf, Performing Antiquity: Ancient Greek Music and Dance from Paris to Delphi, 1890-1930, Oxford, Oxford University Press, 2018, p. 81 et 92.

7. Dorf, 2018, cité n. 6, p. 82-94 (p. 83 pour la citation).
8. Tyler Jo Smith, « Reception or Deception? Approaching Dance through Greek Vase-Painting ", dans Fiona Maclntosh (dir.), The Ancient Dancer in the Modern World: Responses to Greek and Roman Dance, Oxford, Oxford University Press, 2010, p. 83-89.

9. Voir Frederick G. Naerebout, "In Search of a Dead Rat": The Reception of Ancient Greek Dance in Late Nineteenth-Century Europe and America ", dans Macintosh, 2010, cité n. 8, p. 39-56, en part. p. 50-51 pour le manque de clarté de Duncan sur cette question.

10. Artemis Leontis, Eva Palmer-Silekianos: A Life in Ruins, Princeton, Princeton University Press, 2019.

11. Dorf, 2018, cité n. 6, p. 91.

12. Naerebout, 1997, cité n. 1, p. 60-77; Agnes de Mille, Martha: The Life and Work of Martha Graham, New York, Random House, 1991.

13. Fitzgerald, 2019, cité n. 2.

14. Tyler Jo Smith, « Orchêsis Kallinikos: Lillian B. Lawler's Greek Dance Legacy ", dans Classical World, vol. 113, 2020, p. 197-222. Voir aussi Graham Ley, The Theatricality of Greek Tragedy, Chicago / Londres, University of Chicago Press, 2007, p. 150-167.

15. Voir par exemple «Texts and Images as Sources for the Study of Dance in Ancient Greece ", dans Pharos, vol. 3, 1995, p. 23-40 ; et "Dance», dans Rubina Raja et Jörg Rüpke, A Companion to the Archaeology of Religion in the Ancient World, Oxford, Wiley-Blackwell, 2015, p. 107-119. Pour une bibliographie exhaustive sur la danse gréco-romaine jusqu'à 1997, voir Naerebout, 1997, cité n. 1, p. 114-145.

16. Frank Brommer, «Antike Tänze », dans Archäologischer Anzeiger, 1989, p. 483-496.

17. Steven H. Lonsdale, Dance and Ritual Play in Greek Religion, Baltimore, Johns Hopkins University Press, 1993, p. 9-16.

18. Paulette Ghiron-Bistagne, Recherches sur les acteurs dans la Grèce antique, Paris, Les Belles Lettres, 1976, p. 207-297. Voir aussi, par exemple, Arthur D. Trendall et Thomas B.L. Webster, Illustrations of Greek Drama, Londres, Phaidon, 1971.

19. Jean-Claude Poursat, «Les représentations de danse armée dans la céramique attique », dans Bulletin de Correspondance Hellénique, vol. 91, 1967, p. 550-615 ; Marie-Hélène Delavaud-Roux, Les Danses armées en Grèce antique, Aix-en-Provence, Université de Provence, 1993 ; Paola Ceccarelli, La pirrica nell'antichità greco romana: Studi sulla danza armata, Pise / Rome, Istituti Editoriali e Poligrafici Internazionali, 1998.

20. Alkis Raftis, The World of Greek Dance, Athènes,

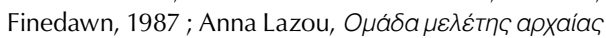

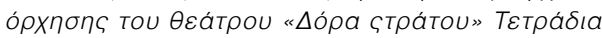
Epyaciac 2013-2018, Athènes, Université d'Athènes, 2019. Voir également Marie-Hélène Delavaud-Roux, "Du nouveau sur la danse et la musique en Grèce ancienne », dans Connaissance Hellénique [en ligne, URL : https://ch.hypotheses.org/3974].

21. Marie-Hélène Delavaud-Roux (dir.), Musiques et danses dans l'Antiquité, Rennes, Presses universitaires de Rennes, 2011 ; Kathryn Soar et Christina Aamont (dir.), Archaeological Approaches to Dance Performance, 
Oxford, BAR, 2014 ; Laura Gianvittorio (dir.), Choreutika: Performing and Theorising Dance in Ancient Greece, Pise et Rome, Fabrizio Serra Editore, 2017 ; Greek and Roman Musical Studies, vol. 8, 2020 (numéro spécial "Music and Materiality »).

22. Voir par exemple Deborah Steiner, "Pot Bellies: The Komast Vases and Contemporary Song ", dans Dimitrios Yatromanolakis, An Archaeology of Representations: Ancient Greek Vase-Painting and Contemporary Methodologies, Athènes, Kardamitsa, 2009, p. 240-281 ; Smith, 2010, cité n. 3 ; Angela Ziskowski, « Clubfeet and Kypselids: Contextualising Corinthian Padded Dancers in the Archaic Period ", dans Annual of the British School at Athens, vol. 107, 2012, p. 211-232 ; Detlev Wannagat, Archaisches Lachen: Die Entstehung einer komischen Bilderwelt in der korinthischen Vasenmalerei, Berlin, De Gruyter, 2015.

23. Tyler Jo Smith, "Komastai or "Hephaistoi": Visions of Comic Parody in Archaic Greece ", dans Bulletin of the Institute of Classical Studies, vol. 52, 2009, p. 69-92 ; Ziskowski, 2012, cité n. 22 ; Wannagat, 2015, cité n. 22 ; et Edith Hall, « Hephaestus the Hobbling Humorist: the Club-Footed God in the History of Early Greek Comedy ", dans Illinois Classical Studies, vol. 43, 2018, p. 366-387.

24. Voir par exemple Ghiron-Bistagne, 1976, cité n. 18 ; Thomas C. Carpenter, Dionysian Imagery in Archaic Greek Art, Oxford, Clarendon Press, 1986 ; François Lissarrague, Un flot d'images : une esthétique du banquet grec, Paris, Adam Biro, 1987 ; Cornelia Isler-Kérenyi, Dionysos nella Grecia arcaica. Il contributo delle immagini, Pise / Rome, Istituti Editoriali e Poligrafici Internazionali, 2001 (édition révisée : Dionysos in Archaic Greece: An Understanding through Images, Leiden, Brill, 2006); Richard Green, " Art and the Theatre in the Ancient World ", dans Marianne McDonald et J. Michael Walton (dir.), The Cambridge Companion to Greek and Roman Theatre, Cambridge, Cambridge University Press, 2007, p. 163-183 ; Eric Csapo et Margaret C. Miller (dir.), The Origins of Theater in Ancient Greece and Beyond: From Ritual to Drama, Cambridge, Cambridge University Press, 2008.

25. Guy M. Hedreen, Silens in Attic Black-figure VasePainting, Ann Arbor, University of Michigan Press, 1992 ; Margaret C. Miller, "Reexamining Transvestism in Archaic and Classical Athens: The Zewadski Stamnos ", American Journal of Archaeology, vol. 103, 1999, p. 223253 ; Eric Csapo, "Dolphins of Dionysus », dans Eric Csapo et Margaret C. Miller (dir.), Poetry, Theory, Praxis, Oxford, Oxbow Books, 2003, p. 69-98; Kenneth S. Rothwell, Nature, Culture, and the Origins of Greek Comedy: A Study of Animal Choruses, Cambridge, CUP, 2007 ; Bernd Seidensticker, " Dance in Satyr Play », dans Oliver Taplin et Rosie Wyles (dir.), The Pronomos Vase and its Context, Oxford, Oxford University Press, 2010, p. 213-229 ; Fabienne Coudin, «Culte et identité culturelle : les offrandes de vases dans les sanctuaires laconiens ", dans François Quantin (dir.), Archéologie des religions antiques, Pau, Université de Pau et des pays de I'Adour, 2011, p. 87-101 ; François Lissarrague, La Cité des satyres : une anthropologie ludique (Athènes, VIe$V^{e}$ siècles avant J.-C.), Paris, Éditions de l'EHESS, 2013 ; Adrien Delahaye, "Les satyres laconiens à l'aune du modèle attique ", dans Kentron, vol. 32, 2016, p. 59-84.
26. Elizabeth W. Barber, The Dancing Goddesses: Folklore, Archaeology, and the Origins of European Dance, New York, Norton, 2013, ch. X, tient compte des deux catégories.

27. Voir par exemple Elizabeth G. Pemberton, «Wine, Women and Song: Gender Roles in Corinthian Cult ", dans Kernos, vol. 12, 2000, p. 85-106 ; Lesley A. Beaumont, Childhood in Ancient Athens: Iconography and Social History, Londres, Routledge, 2012, p. 149-152.

28. Marie-Christine Villanueva Puig, Ménades : Recherches sur la genèse iconographique du thiase féminin de Dionysos des origines à la fin de la période archaïque, Paris, Les Belles Lettres, 2009 ; Eleni Manakidou, Frauentänze für Dionysos in der spätarchaischen Vasenmalerei Athens, Kilchberg, Akanthus, 2017.

29. Sarah Peirce, "Visual Language and Concepts of Cult on the "Lenaia Vases" ", dans Classical Antiquity, vol. 17, 1998, p. 59-95.

30. Voir Frederick G. Naerebout, "Moving Events. Dance at Public Events in the Ancient Greek World: Thinking through its Implications ", dans Eftychia Stavrianopoulou (dir.), Ritual and Communication in the Graeco-Roman World, Liège, Presses universitaires de Liège, 2006, p. 49.

31. Voir par exemple Sonia Alconini, Rito, símbolo e historia en la pirámide de Akapana, Tiwanaku: un análisis de cerámica ceremonial prehispánica, La Paz, Editorial Acción, 1995.

32. Je m'oppose ici à l'opinion exprimée par Marianne MacDonald, « Dancing Drama: Ancient Greek Theater in Modern Shoes and Shows ", dans Nadine George-Graves (dir.), The Oxford Handbook of Dance and Theater, Oxford, Oxford University Press, 2015, p. 280, qui considère les vases comme des sources muettes ( $~ e v i$ dence [...] derived from mute sources such as vases »).

33. Voir Laura Gianvittorio-Ungar, "Narratives in Motion: the Art of Dancing Stories in Antiquity and Beyond. Report on an Interdisciplinary Symposium with Scholars and Performers ", dans Greek and Roman Musical Studies, vol. 8, 2020, p. 174-189. 